\title{
Estrutura trófica das aves da Cidade Universitária de Dourados - Mato Grosso do Sul
}

\section{Trophic structure of birds of the University City of Dourados - Mato Grosso do Sul}

\author{
1 Universidade Federal da Grande Dourados (UFGD), Dourados, \\ MS, Brasil \\ ${ }^{2}$ Universidade Estadual de Mato Grosso do Sul (UEMS), Dourados, \\ MS, Brasil
}

* Correspondência: rafaelgoes@ufgd.edu.br

Submetido: 7 abr 2021 | Aprovado: 10 set 2021

DOI: http://dx.doi.org/10.7213/acad.2021.19010

Rev. Acad. Ciênc. Anim. 2021;19:e19010

Resumo

O presente trabalho teve como objetivo avaliar a estrutura trófica da avifauna da Cidade Universitária de Dourados, MS, composta pela Universidade Federal da Grande Dourados (UFGD) e Universidade Estadual de Mato Grosso do Sul (UEMS). Entre os anos de 2017 e 2019 foram identificadas 142 espécies alocadas em um total de 43 famílias, com destaque para as famílias Tyrannidae (19) e Thaupidae (16). Considerando as estruturas tróficas, as análises de correspondências múltiplas demonstraram que as aves onívoras (28,25\%) e granívoras $(26,70 \%)$ representaram a maior parte das contribuições, totalizando 54,95\%. Pela análise de Cluster comprovou-se a representação das estruturas tróficas, com destaque para o grupo trófico de onívoros
Rafael Henrique de Tonissi e Buschinelli de Goes (D) $^{1 *}$ Leonardo de Oliveira Seno (1) ${ }^{1}$ Mayara Cáceres $\mathbb{D}^{2}$ e granívoros, seguidos pelos insetívoros e frugívoros. A sazonalidade e a disponibilidade de alimento influencia a composição dos grupos tróficos, destacando-se na Cidade Universitária os grupos onívoros e granívoros. O grau de urbanização apresentado pelo câmpus universitário relaciona-se à maior ocorrência de espécies generalistas.

Palavras-chave: Avifauna. Observação de aves. Ornitologia.

\section{Abstract}

The present work aimed to evaluate the avifauna trophic structure in the City University of Dourados, MS, composed of the universities Universidade Federal da Grande Dourados (UFGD) and Universidade Estadual de Mato Grosso do Sul (UEMS). Between the years of 2017 and 2019, 142 species from 43 families were identified, with emphasis on the families Tyrannidae (19) and Thaupidae (16). Considering the trophic structures, multiple correspondence analysis showed that omnivorous birds (28.25\%) and granivorous birds (26.70\%) represented the most of the contributions, totaling 54.95\%. Cluster analysis demonstrated the representation of trophic structures, highlighting the trophic group of omnivores and granivores, followed by insectivores and fruit-eaters. Seasonally and availability 
of food influence the composition of the trophic groups, with the omnivorous and granivorous groups standing out in the City University. The urbanization degree presented by the university campus is related to the increase in generalist species occurences.

Keywords: Avifauna. Birdwatching. Ornithology.

\section{Introdução}

O conhecimento sobre as aves brasileiras começou a ser estabelecido por meio de dados originários de estudos de natureza regional (Pacheco e Bauer, 2001). Por várias razões, o Mato Grosso do Sul apenas recentemente iniciou o inventário organizado de sua avifauna, contrastando fortemente com outros estados brasileiros (Nunes et al., 2017).

Apesar disso, o ambiente urbano fornece informações relevantes sobre o comportamento e estrutura da comunidade de aves, em função da fragmentação e alteração das vegetações (Matarazzo-Neuberger, 1995). Aves são o grupo animal mais estudado em ambientes urbanos (Turner, 2003), tornando-se importante para subsidiar informações para a preservação de habitats, constatação do surgimento de espécies sinantrópicas ou o desaparecimento de espécies incapazes de se adaptar aos habitats transformados (MatarazzoNeuberger, 1995).

O levantamento da avifauna e o agrupamento trófico servem para identificar a complexidade estrutural de habitats e auxiliar na identificação do grau de preservação (Scherer et al., 2005). Destaca-se que o levantamento das espécies locais e migratórias sofre alteração com base em mudanças estruturais, sejam elas naturais ou provocadas por atividades humanas (Aleixo, 1999).

As modificações ambientais podem ser detectadas por meio da estrutura trófica (Catian et al., 2011). Espécies que incluem uma diversidade de itens alimentares, como as onívoras, ou espécies insetívoras generalistas são capazes de explorar diversos tipos de habitats e tornam-se beneficiadas em ambientes alterados (Willis, 1979; Santos, 2004). Já espécies especialistas, como frugívoras e insetívoras, dependem de uma grande disponibilidade de alimento ao longo do ano, sendo vulneráveis a ambientes mais impactados (Willis, 1979; Motta Jr, 1990).

Os câmpus universitários geralmente apresentam considerável porção de área de mata, muitas vezes fragmentada, porém utilizada como refúgio ou até mesmo como habitat (Lopes e Anjos, 2006). A Cidade Universitária de Dourados é caracterizada por uma flora diversificada e uma grande diversidade de aves. Foram observadas 72 espécies no câmpus da Universidade Federal da Grande Dourados (UFGD), sendo essas distribuídas em 21 famílias e 10 ordens, em que o maior número de representantes foram de Emberizidae e Tyrannide (Catian e Aranda, 2009; Catian et al., 2011).

O presente trabalho, portanto, teve como objetivo estudar a estrutura trófica das espécies de aves que frequentam a Cidade Universitária de Dourados, composta pelos câmpus da Universidade Federal da Grande Dourados (UFGD) e da Universidade Estadual de Mato Grosso do Sul (UEMS), bem como seus arredores.

\section{Material e métodos}

A Cidade Universitária de Dourados está localizada no município de Dourados, ao sul do Mato Grosso do Sul, compreendida pelas coordenadas geográficas $22^{\circ} 13^{\prime} 16^{\prime \prime} \mathrm{S}$ e $54^{\circ} 48^{\prime} 20^{\prime \prime} \mathrm{W}$. Cerca de $430 \mathrm{~m}$ acima do nível do mar, localiza-se a $10 \mathrm{~km}$ da área urbana de Dourados, próxima (cerca de $2 \mathrm{~km}$ ) a um fragmento de mata nativa (Mata do Azulão), de várias áreas de plantio de cultivos sazonais e de área de pastagem. Possui uma área total de 90 hectares e apresenta solo do tipo latossolo vermelho (Embrapa, 1999). O clima da região é classificado como mesotérmico úmido (Cfa) e tropical úmido (Aw), com estação chuvosa no verão e seca no inverno (Koeppen, 1948).

Mesmo sendo enquadrada como um ambiente urbano, a cidade universitária apresenta uma caracterização florística diversificada, contendo espécies nativas, áreas com plantio, horta de plantas medicinais, pomar com cultivares comerciais, além de eucaliptos e pinheiros (Catian et al., 2011).

As observações ocorreram no período de janeiro a dezembro dos anos 2017, 2018 e 2019. Os registros foram realizados semanalmente pela manhã (6h às $11 \mathrm{~h}$ ) e à tarde (13h às $17 \mathrm{~h})$, com períodos de 30 min entre cada observação, tendo em sua totalidade 
um período de 65 horas de observação/ano. Conforme apresentado na Figura 1, dentre os locais de observação foram determinadas seis áreas com maior concentração de aves: UEMS, auditório, blocos de aula, horta, área experimental e Faculdade de Engenharia (FAEN).

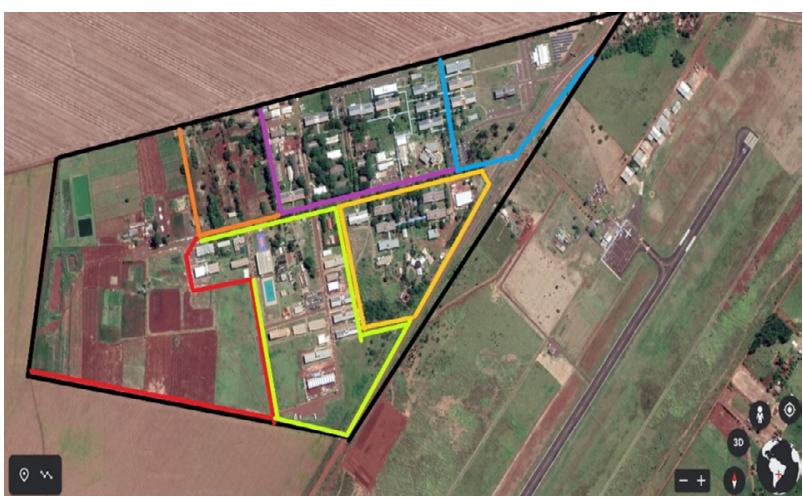

Figura 1 - Foto ilustrativa da Cidade Universitária de Dourados (dezembro de 2020, Google Earth).

Nota: Universidade Estadual de Mato Grosso do Sul (UEMS) (Amarelo Escuro); Auditório (Azul); Blocos de aula (Roxo); Horta (Laranja); Área experimental (Vermelho); Faculdade de Engenharia (Amarelo Claro).

Utilizou-se para as observações o método do trajeto irregular, no qual cada ave que pudesse ser identificada, visualmente ou pela voz, era registrada apenas durante o tempo de caminhada pelo ponto amostral. $O$ registro foi realizado por meio de observação em transectos de $50 \mathrm{~m}$, onde foram catalogados todos os indivíduos encontrados através de identificação por reconhecimento visual (Nascimento et al., 2005; Scherer et al., 2005), com o auxílio de guia de campo (De la Peña e Rumboll, 2001; Souza, 2004), além dos padrões de vocalização e outras manifestações sonoras (estalidos e batidas de asas). A identificação das espécies foi obtida conforme Sick (2001); a classificação taxonômica e a nomenclatura científica seguiram o Comitê Brasileiro de Registros Ornitológicos (Piacentini et al., 2015; Pacheco et al., 2021). Todas as aves foram fotografadas com máquina digital (Canon T5i, lente Sigma 150-600 mm).

Foram avaliadas as áreas: auditório (área 1), UEMS (área 2) e horta/pomar (área 3), com predominância de espécies frutíferas das famílias Anacardiaceae
R. Br., Araliaceae Juss., Fabaceae Lindl., Lauraceae Juss., Malpighiaceae Juss., Malvaceae Juss., Moraceae Gaudich., Musaceae Juss., Myrtaceae Juss., Rubiaceae Juss., Rutaceae Juss., Vitaceae Juss., além de Pinaceae Spreng. ex Rudolphi; horta (área 3) com espécies medicinais e plantações de hortaliças, além de espécies de Annonaceae Juss., Caricaceae Dumort., Fabaceae, Moraceae, Myrtaceae, Poaceae Barnhart e Rosaceae Juss. Na área 1, verificou-se ainda a presença de Eucalyptus, Pinus e Bignoniaceae. Os blocos de aula (área 4) e a FAEN (área 6) apresentam predominância de Araliaceae (Mandiocão), Bignoniaceae Juss. (Ipê), Malvaceae (Paineira), Myrtaceae (Eucalipto) e Pinaceae (Pinheiro, Cedro), enquanto a área experimental (área 5) é composta por áreas agrícolas (predominância de milho e soja) e de pastagens (Brachiaria sp.)

As aves registradas foram caracterizadas segundo dados da literatura (Gimenes e Anjos, 2000; Catian e Aranda, 2009; Catian et al., 2011) para seus grupos tróficos: carnívoras (C), alimentam-se principalmente de vertebrados pequenos; insetívoras (I), alimentação majoritariamente composta por insetos; frugívoras $(F)$, alimentação através de frutas; onívoras $(\mathrm{O})$, dieta rica em insetos, frutos e pequenos invertebrados; granívoras $(\mathrm{G})$, alimentam-se de sementes; e nectarívoras $(\mathrm{N})$, alimentam-se exclusivamente de néctar. Analisouse a frequência cumulativa de acordo com o grupo trófico e a análise de Cluster em relação às espécies e grupos tróficos. Os grupos tróficos foram avaliados por análise de correspondências múltiplas (Bendixen, 1995), utilizando o software estatístico R.

\section{Resultados}

Durante o período de observação foram identificadas 142 espécies de um total de 43 famílias e 22 ordens (Tabela 1). Tyrannidae e Thraupidae foram as famílias que apresentaram maior número de espécies, 19 e 16 respectivamente.

Considerando as estruturas tróficas, as análises de correspondências múltiplas apresentaram respostas para as dimensões 1 e 2 (Figuras 2 - 4), destacando que aves onívoras $(28,25 \%)$ e granívoras $(26,70 \%)$ representaram a maior parte das contribuições, totalizando 54,95\%. Pela análise de Cluster (Figura 5), comprova-se a representação das estruturas tróficas avaliadas, destacando que os grupos tróficos onívoros e granívoros foram os mais frequentes, seguidos pelos insetívoros e frugívoros. 


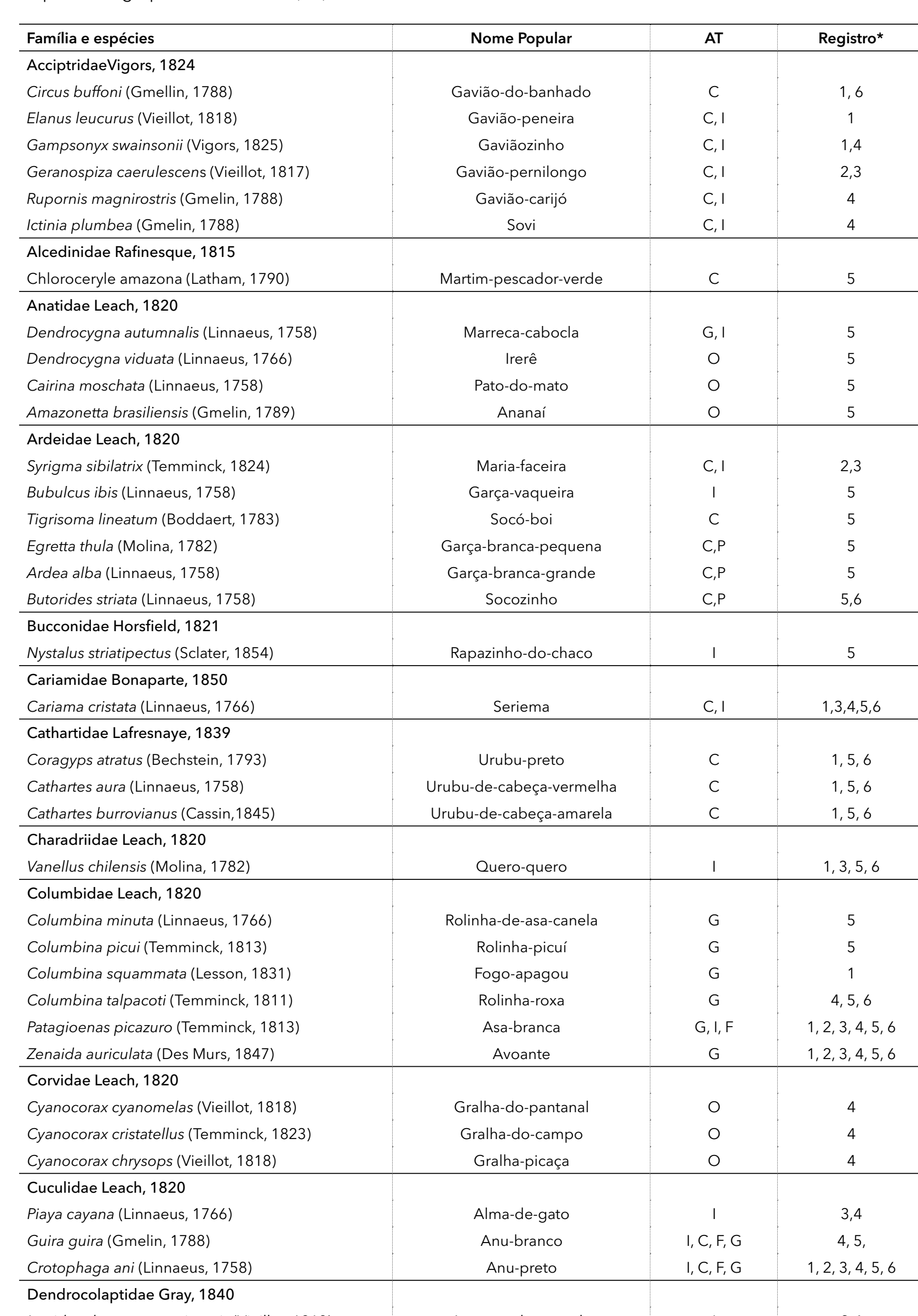

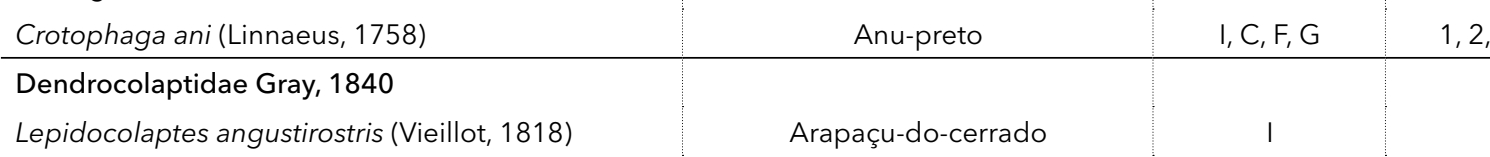

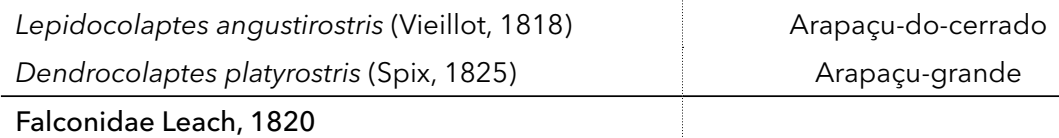

\begin{tabular}{|c|}
\hline 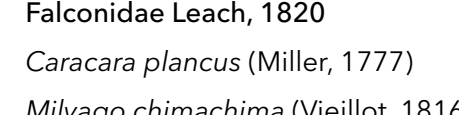 \\
\hline 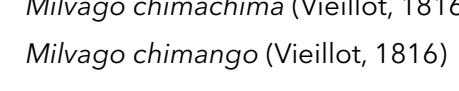 \\
\hline
\end{tabular}

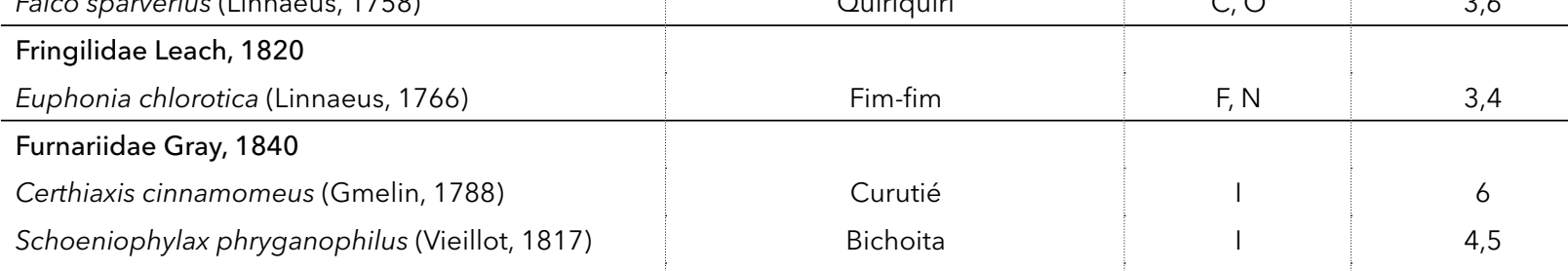

\begin{tabular}{|c|c|c|c|}
\hline 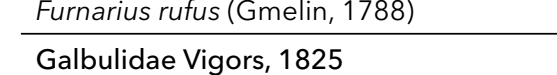 & & & \\
\hline 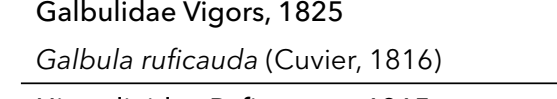 & Ariramba-de.cauda-riva & 1 & 4 \\
\hline 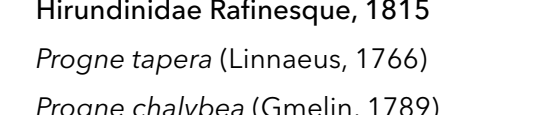 & Andorinh adocampo & 1 & $1,2,3,4,5,6.6$ \\
\hline 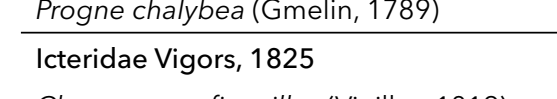 & & & \\
\hline 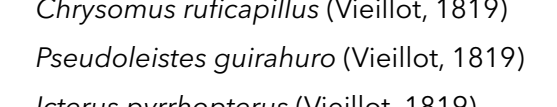 & 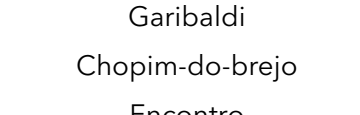 & $\begin{array}{c}F, G, 1 \\
G \\
G\end{array}$ & $\begin{array}{c}5 \\
6 \\
\end{array}$ \\
\hline 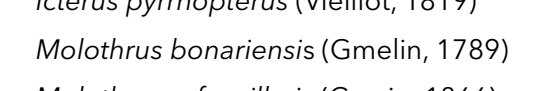 & 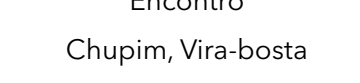 & & \\
\hline 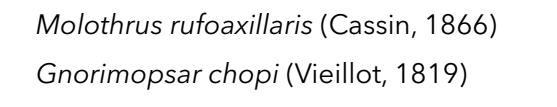 & $\begin{array}{l}\text { Chupimazzeviche } \\
\text { passare-preto }\end{array}$ & $\begin{array}{l}\circ \\
0\end{array}$ & ${ }_{3}^{3}$ \\
\hline 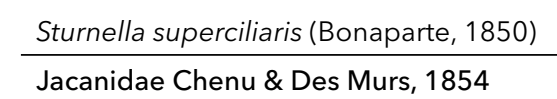 & Policiainglestadosulu & & \\
\hline 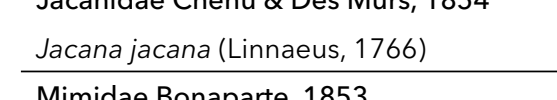 & Jasana & $\circ$ & 5 \\
\hline 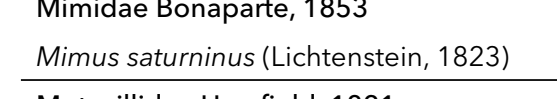 & Sabiä.do-campo & $\circ$ & $2,3,4,5$ \\
\hline 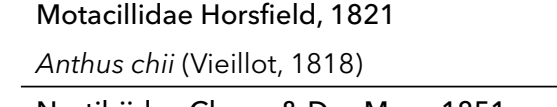 & Caminheriozumbidor & 0 & 5 \\
\hline 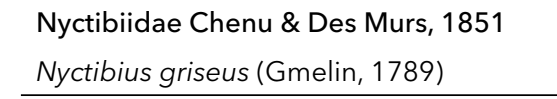 & Unutau & 1 & 3 \\
\hline
\end{tabular}

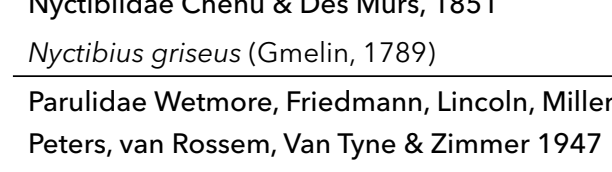

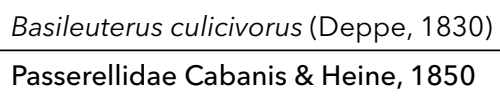

\begin{tabular}{llll} 
Ticotico-docampo & 6 & 5 \\
\hline
\end{tabular}

Passerd donsticicus 1 in

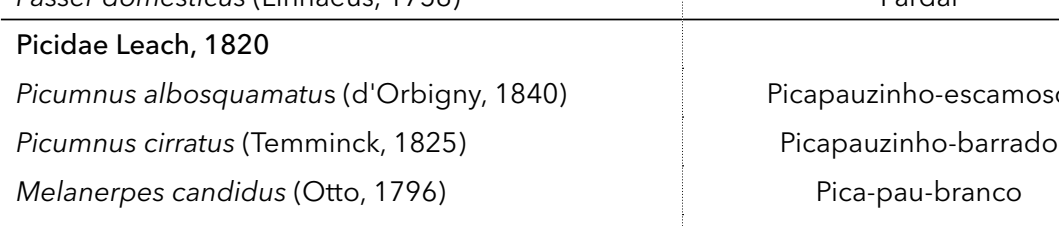

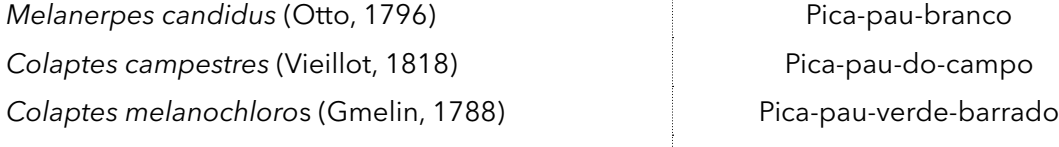

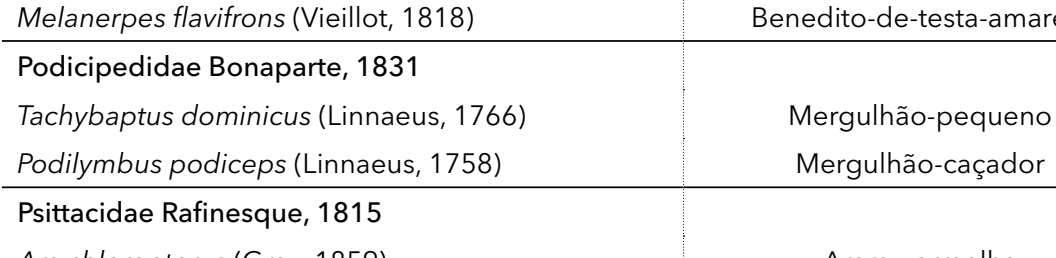

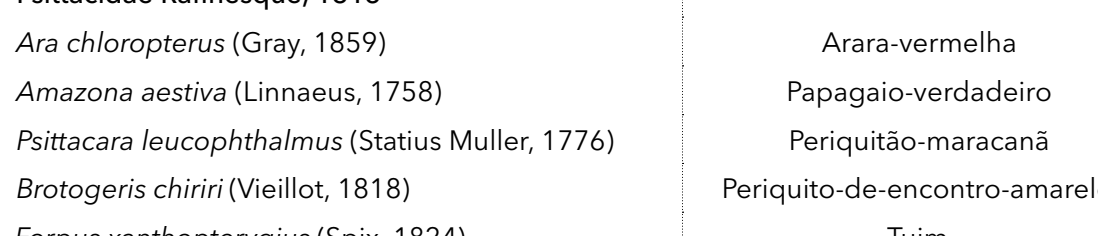

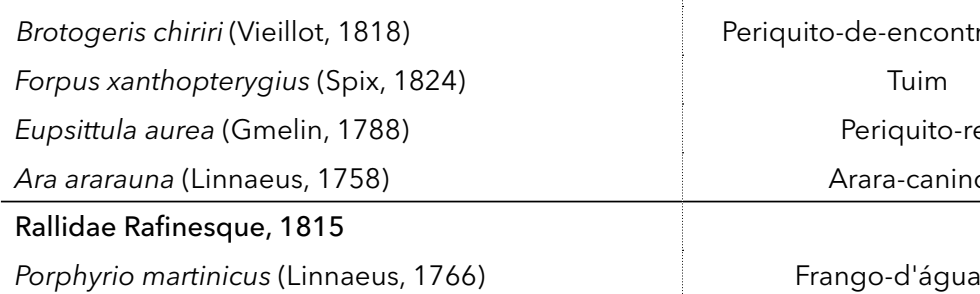

o.d'́gua azul

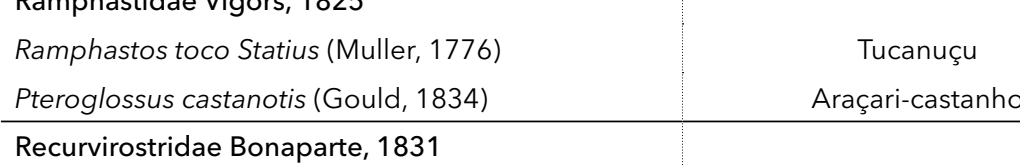

\begin{tabular}{l|l|l|}
\hline & & 3 \\
\hline
\end{tabular}

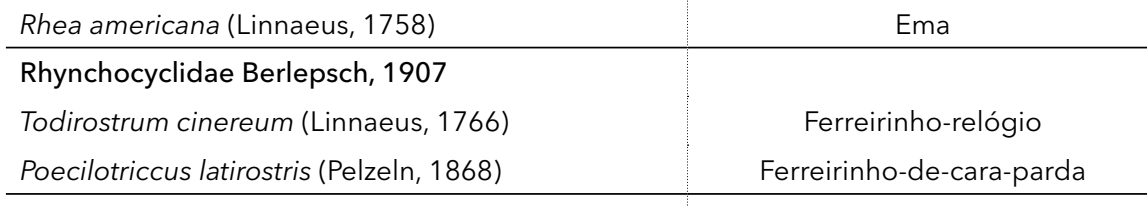

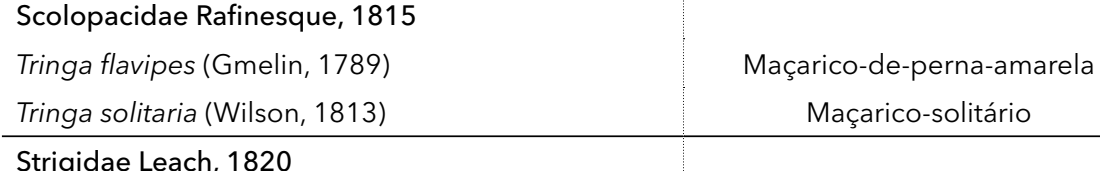

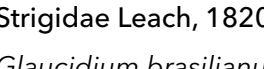

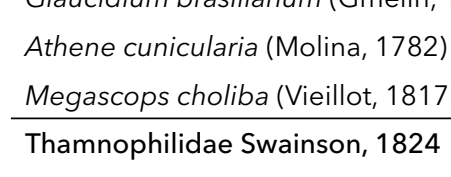

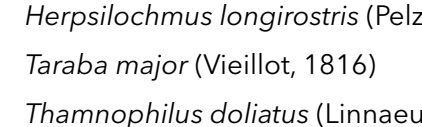

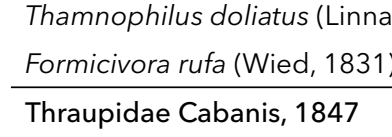

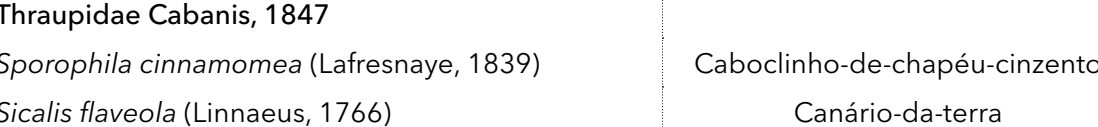

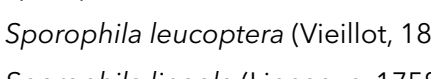

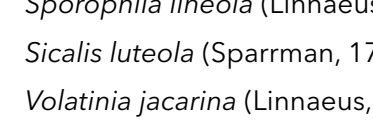

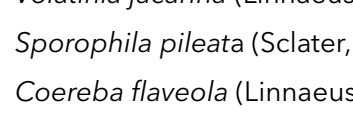

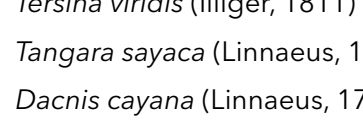

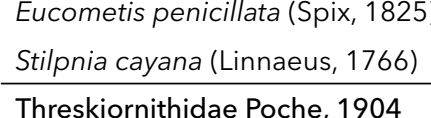

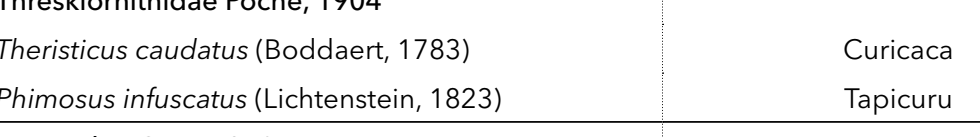

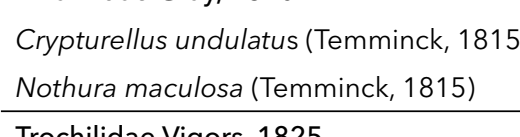

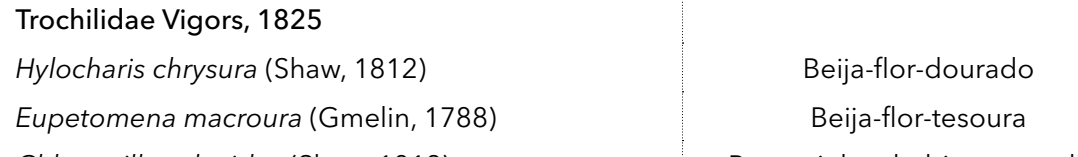

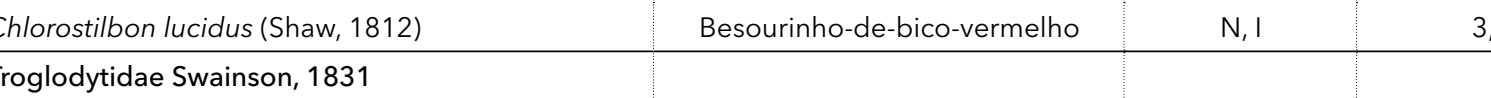

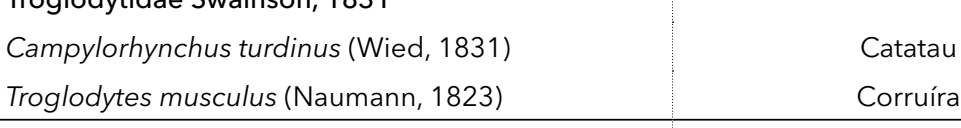

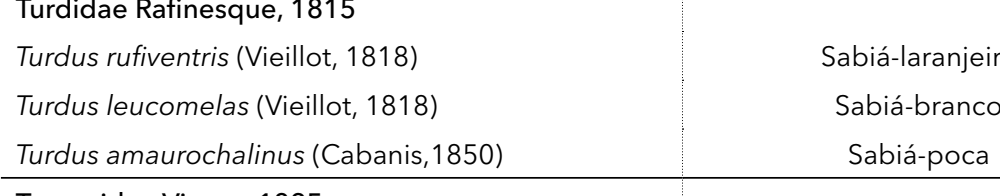

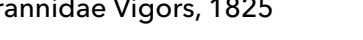

Solob pocter

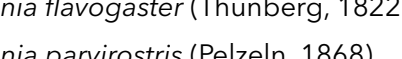

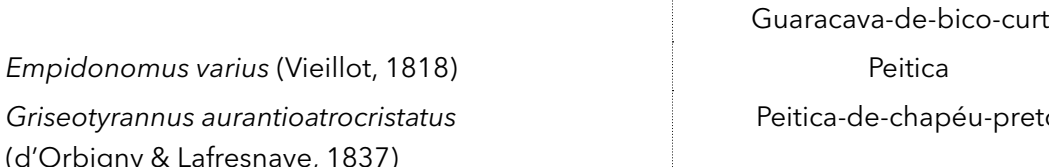

Wherentets y yetapa (Villot,

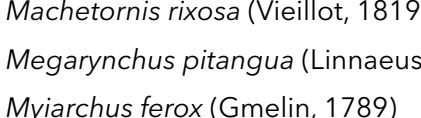

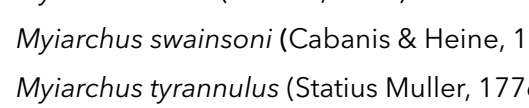

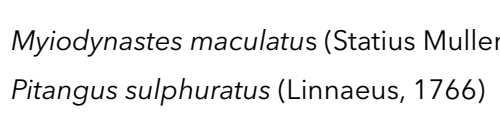

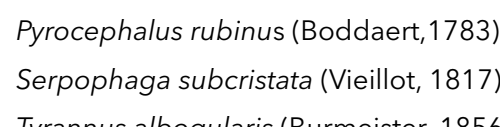

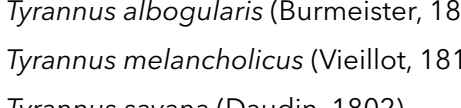

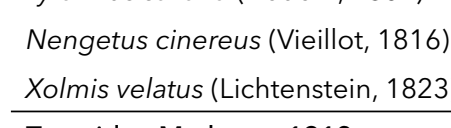

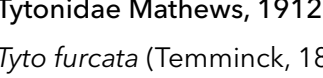

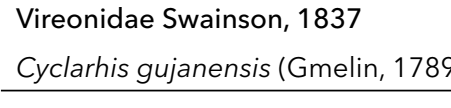

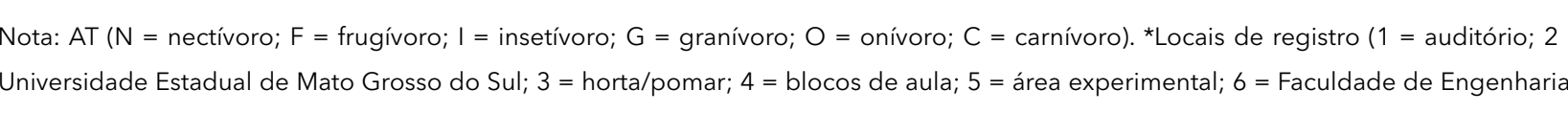




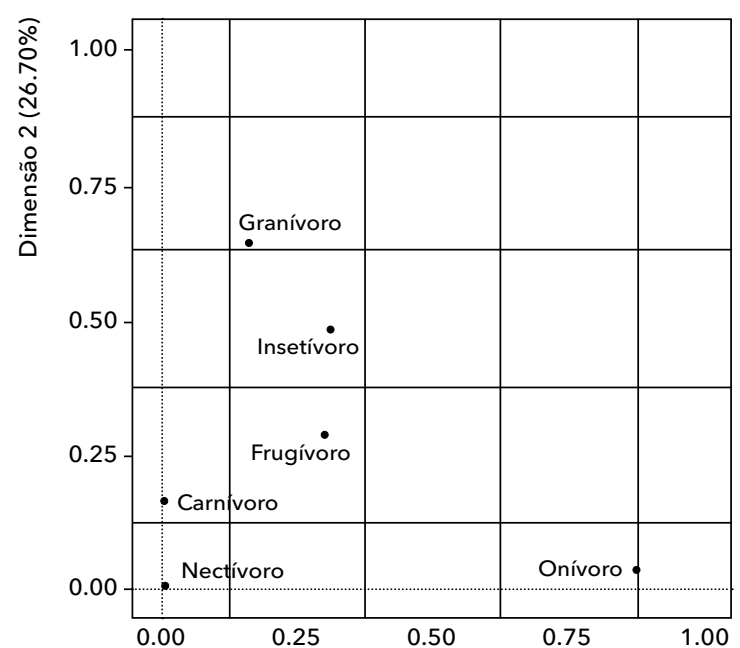

Dimensão 1 (28.25\%)

Figura 2 - Representação das variáveis para estrutura trófica das aves observadas, de acordo com as análises de correspondências múltiplas.

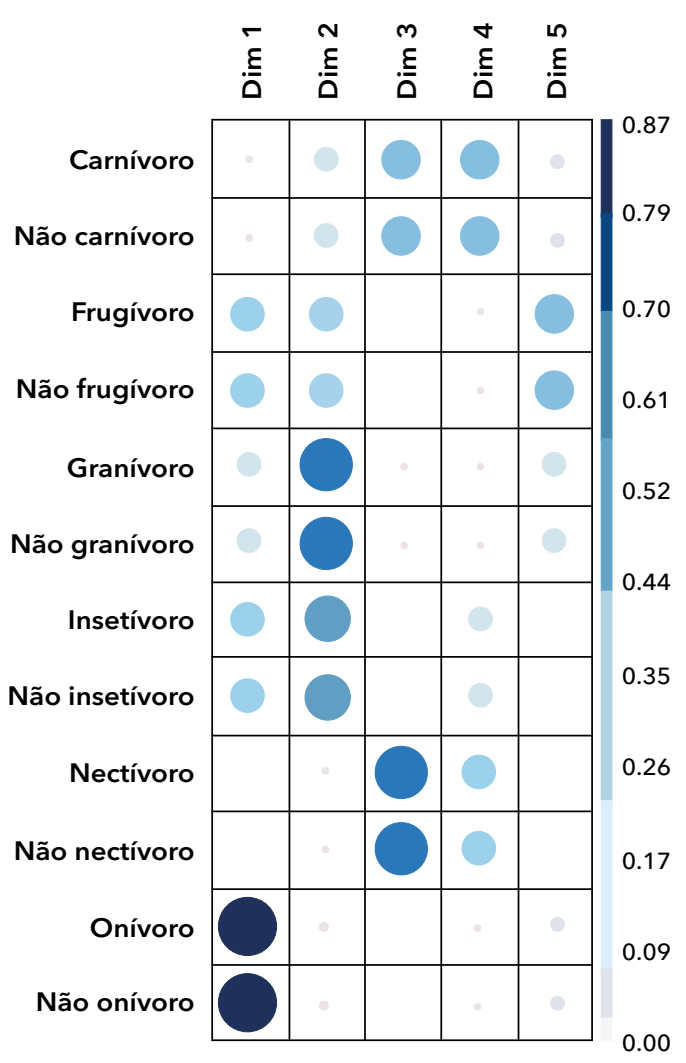

Figura 3 - Representação das estruturas tróficas por dimensões (Dim) de acordo com as análises de correspondências múltiplas.

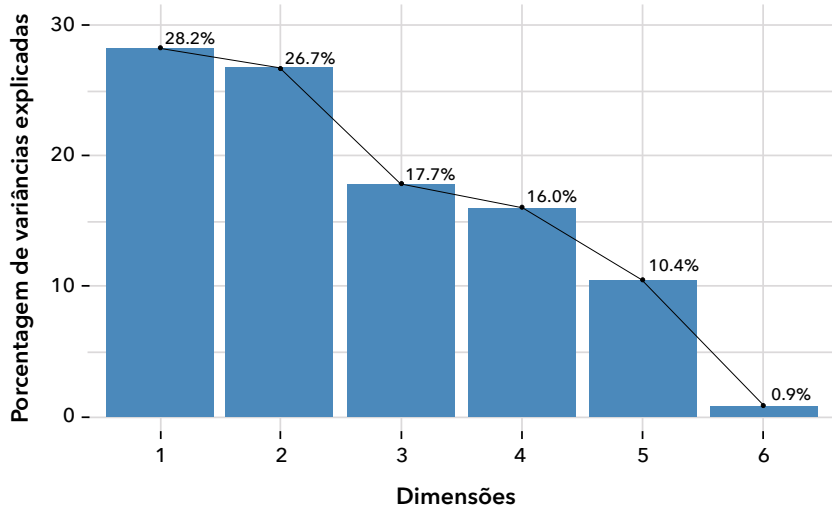

Figura 4 - Percentagem para a explicação das variáveis de acordo com as dimensões conforme análises de correspondências múltiplas.

Dendrograma

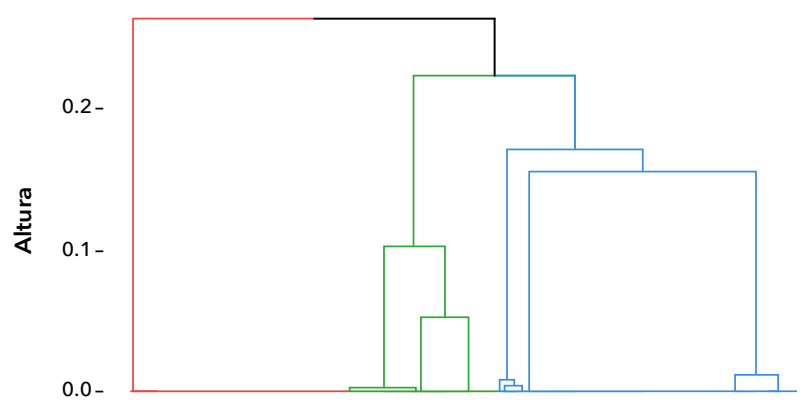

Figura 5 - Diagrama resultante da análise de agrupamento (cluster) entre as variáveis avaliadas em relação às aves observadas e grupos tróficos.

\section{Discussão}

O maior número de aves de grupos tróficos menos especializados, como os onívoros, pode ser um indicador de perturbações ambientais, sendo estas espécies capazes de explorar diferentes tipos de habitats e incluírem alimentação variável (Motta Jr, 1990), fato confirmado pela abundância de espécies desses grupos identificadas neste trabalho. A onivoria apresenta efeito tampão contra flutuações alimentares, o que pode estar associado ao aumento dessas espécies generalistas em áreas de vegetação secundária, exótica ou antropizadas (Willis, 1976). 
Quando se considera o número de registros ou de indivíduos para a estruturação das relações tróficas há uma nítida relação entre os tipos de dieta e o habitat, destacando, assim, o grupo de onívoros (Figuras 3 e 4). A maior disponibilidade de alimentos encontrada entre as áreas de pomar/horta e bloco de aulas pode ter favorecido a maior presença dos grupos tróficos onívoros e insetívoros. Já para a área de pomar/horta e área experimental, a presença de maior parte de grãos cultivados tem favorecido a presença do grupo granívoro.

Catian et al. (2011), avaliando a estrutura trófica de aves no câmpus da UFGD, exaltaram a presença de árvores frutíferas no pomar, hortaliças em campo aberto e herbáceas e arbustos na horta, o que proporciona uma maior abundância de insetos, bem como a presença de sementes provenientes de árvores nativas e exóticas entre os blocos de aula, o que contribuiu para identificar a predominância dos grupos onívoros e insetívoros (Catian e Aranda, 2009; Catian at al., 2011).

Nos estudos de Catian e Aranda (2009) e Catian at al. (2011) foram identificadas apenas 72 espécies de um total de 25 famílias, números esses inferiores aos obtidos neste trabalho, onde foram identificadas 43 famílias de um total de 142 espécies observadas. Bomediano (2004) identificou 102 espécies, com esforço amostral de 100 h, na Mata do Azulão, região localizada nos arredores da cidade universitária. Catian e Aranda (2009) destacam que $45 \%$ das espécies identificadas na UFGD são observadas também na Mata do Azulão, indicando que o câmpus universitário pode ser uma alternativa às espécies para a aquisição de recursos alimentares adicionais.

O aparecimento das espécies ao longo dos anos foi gradativo, conforme o florescimento e produção de frutos entre os meses de maio e agosto. Diferentes espécies possuem hábitos alimentares semelhantes, ocorrendo maior diferença entre as áreas estudadas, o que demonstra que diferentes espécies podem atuar de forma similar entre os ambientes.

Apesar de os frugívoros estarem enquadrados na dimensão 5 (Figura 3), neste trabalho com apenas 10,4\% dos efeitos observados (Figura 4), as aves deste grupo tendem a tornar-se nômades, decorrente da abundância, conspicuidade e distribuição territorial e espacial dos frutos (Poulin et al., 1994). De acordo com Willis (1979) e Anjos (2001), os frugívoros apresentam alta capacidade de deslocamento, podendo procurar alimento em outros fragmentos, desde que não muito distantes, e podendo se mover frequentemente entre manchas florestais mais do que as espécies insetívoras e onívoras.

O grau de frugivoria pode variar ao longo do ano em uma mesma espécie de ave, como ocorre com o gênero Turdus spp. e outras espécies que se apresentam como frugívoras durante o fim da estação seca, favorecendo o acúmulo de gordura para migração, ou conforme a disponibilidade de alimentos (Catian et al., 2011).

Uma vez que os hábitos alimentares são influenciados pelo deslocamento das aves, espécies insetívoras tendem a ser favorecidas em decorrência da abundância de insetos e de sua distribuição espacial relativamente uniforme (Piratelli e Pereira, 2002). Os impactos decorrentes da alteração da cobertura vegetal, com predominânicia de lavouras, reduz o tamanho das populações, além da colonização por novas espécies, aumentando a competição e predação e reduzindo a biodiversidade (Catian e Aranda, 2009). Esse efeito pôde ser visto na área experimental, o que apresentou maior efeito para aves granívoras em detrimento das insetívoras.

Ambientes urbanos fragmentados apresentam predominância de grupos insetívoros e onívoros (Galina e Gimenes, 2006), o que está de acordo com a expansão urbana apresentada pela Cidade Universitária de Dourados nos últimos dez anos. Esse ambiente urbanizado contrasta com habitats mais complexos, que tendem a sofrer menores variações em seus recursos, mantendo, portanto, a diversidade de sua avifauna (Negret, 1983).

Segundo Catian et al. (2011), a obtenção de recursos alimentares pelas aves difere entre as espécies e entre os micro-habitats, como pode ser observado nas famílias Picidae (pica-paus) e Dendrocolaptidae (arapaçus), que possuem seus habitats específicos nos troncos ocos de árvores secas. Willis (1979) cita as aves insetívoras escaladoras de troncos e galhos como as mais rapidamente extintas diante da alteração de seu ambiente. Assim, cada vez mais aves onívoras, e possivelmente insetívoras menos especializadas, terão menor predominância, o que não ocorreria para frugívoras (Silva, 1986) ou insetívoras mais especializadas (Willis, 1979).

Das aves de topo de cadeia alimentar, foram registradas seis espécies da família Accipitridae e 
quatro da família Falconidae. Esses números são maiores dos que os encontrados por Catian et al., (2011), possivelmente devido ao aumento da área amostral, que acarretou aumento no número de indivíduos (Gimenes e Anjos, 2000, 2003).

Devido à sazonalidade de alimento e à composição dos grupos tróficos, constata-se que a comunidade de aves da Cidade Universitária de Dourados está estruturada competitivamente, com as espécies competindo por recursos alimentares.

\section{Conclusão}

A flutuação e a disponibilidade de alimento influenciam na composição dos grupos tróficos, destacando-se na Cidade Universitária de Dourados os grupos onívoros e granívoros.

O grau de urbanização apresentado pelo câmpus universitário está relacionado ao maior número de espécies generalistas. $O$ ambiente em que estes indivíduos foram localizados, ainda que em perímetro urbano, é favorável para a sua permanência. Fazemse necessários estudos contínuos da avifauna a fim de avaliar o efeito do crescimento urbano na biodiversidade da Cidade Universitária de Dourados, no Mato Grosso do Sul.

\section{Referências}

Aleixo A. Effects of selective logging on a bird community in the Brazilian Atlantic Forest. Condor. 1999;101(3):537-48.

Anjos L. Bird communities in five Atlantic Forest fragments in Southern Brazil. Ornitol Neotrop. 2001;12:11-27.

Bendixen MT. Compositional perceptual mapping using chisquared trees analyses and correspondence analyses. J Mark Manag. 1995;11(6):571-81.

Bomediano LM. Avifauna em fragmento de mata semidecídua, Fazenda Azulão, Dourados, MS [monografia]. Dourados: Universidade Federal do Mato Grosso do Sul; 2004.23 p.

Catian G, Aranda R. Utilização do Campus da Universidade Federal da Grande Dourados como refúgio para as aves do fragmento da Mata do Azulão em Dourados, Estado do Mato Grosso do Sul, Brasil. Acta Sci Biol Sci. 2009;31(4):415-9.
Catian G, Fernandes WD, Aranda R. Estrutura trófica de aves diurnas no campus da Universidade Federal da Grande Dourados, MS. Rev Bras Ornitol. 2011;19(3):439-46.

De la Penã MR, Rumboll M. Birds of Southern South America and Antarctica. Princenton, NJ: Princeton University Press; 2001. $304 \mathrm{p}$.

Embrapa - Empresa Brasileira de Pesquisa Agropecuária. Sistema brasileiro de classificação de solos. Brasília: Embrapa Produção de Informação; 1999.

Galina AB, Gimenes MR. Riqueza, composição e distribuição espacial da comunidade de aves em um fragmento florestal urbano em Maringá, norte do estado do Paraná, Brasil. Acta Sci Biol Sci. 2006;28(4):379-88.

Gimenes MR, Anjos L. Distribuição espacial de aves em um fragmento florestal do campus da Universidade Estadual de Londrina, Norte do Paraná, Brasil. Rev Bras Zool. 2000;17(1): 263-71.

Gimenes MR, Anjos L. Efeitos da fragmentação florestal sobre as comunidades de aves. Acta Sci Biol Sci. 2003;25(2): 391-402.

Koeppen W. Climatologia. Buenos Aires: Fondo de Cultura; 1948. p. 152-92.

Lopes EV, Anjos L. A composição da avifauna do campus da UEL, norte do Paraná, Brasil. Rev Bras Zool. 2006;23(1): $145-56$

Matarazzo-Neuberger WM. Comunidades de aves de cinco parques e praças da grande São Paulo, Estado de São Paulo. Ararajuba. 1995;3:13-9.

Motta Jr JC. Estrutura trófica e composição da avifauna de três habitats terrestres na região central do Estado de São Paulo. Ararajuba. 1990;1:65-71.

Nascimento JLX, Sales Jr LG, Sousa AEBA, Minns J. Avaliação rápida das potencialidades ecológicas e econômicas do parque nacional de Ubajara, Ceará, usando aves como bioindicadores. Ornithologia. 2005;1(1):33-42.

Negret AJ. Diversidade e abundância da avifauna da reserva ecológica do IBGE [dissertação]. Brasília: Universidade de Brasília; 1983. 136 p. 
Nunes AP, Straube FC, Laps RR, Posso SR. Checklist das aves do Estado do Mato Grosso do Sul, Brasil. Iheringia Ser Zool. 2017;107(supl.):e2017154.

Pacheco JF, Bauer C. A lista de aves do Espírito Santo de Ruschi (1953): uma análise crítica. In: Albuquerque JLB, Candido Jr JF, Straube FC, Roos AL, editores. Ornitologia e Conservação: da Ciência às Estratégias. Tubarão: Unisul; 2011 p. 261-76.

Pacheco JF, Silveira LF, Aleixo A, Agne CE, Bencke GA, Bravo GA, et al. Annotated checklist of the birds of Brazil by the Brazilian Ornithological Records Committee - second edition. Ornithol Res. 2021;29:94-105.

Piacentini VQ, Aleixo A, Agne CE, Maurício GN, Pacheco JF, Bravo GA, et al. Annotated checklist of the birds of Brazil by the Brazilian Ornithological Records Committee. Rev Bras Ornitol. 2015;23(2):91-298.

Piratelli A, Pereira MR. Dieta de aves na regiao leste de Mato Grosso do Sul, Brasil. Ararajuba. 2002;10(2):131-9.

Poulin B, Lefebvre G, McNeil R. Characteristics of feeding guilds and variation in diets of bird species of three adjacent tropical sites. Biotropica. 1994;26(2):187-97.

Santos AMR. Comunidade de aves em remanescentes florestais secundários de uma área rural no sudeste do Brasil. Ararajuba. 2004;12(1):41-9.
Scherer A, Scherer SB, Bugoni L, Mohr LV, Efe MA, Hartz SM. Estrutura trófica da avifauna em oito parques da cidade de Porto Alegre, RS. Ornithologia. 2005;1(1):25-32.

Sick H. Ornitologia Brasileira. Rio de Janeiro: Editora Nova Fronteira; 2001.862 p.

Silva JMC. Estrutura trófica e distribuição ecológica da avifauna de uma floresta de terra firme na Serra dos Carajás, estado do Pará. Anais do XIII Congresso Brasileiro de Zoologia; 2-7 fev 1986; Cuiabá, MT. Cuiabá: UFMT; 1986. 189 p.

Souza D. Todas as aves do Brasil: guia de campo para identificação. 2 ed. Feira de Santana: Dall; 2004.

Turner WR. Citywide biological monitoring as a tool for ecology and conservation in urban landscapes: the case of the Tucson Bird Count. Landsc Urban Plan. 2003;65(3):149-66.

Willis EO. Effects of a cold wave on an Amazonian avifauna in the upper Paraguay drainage, western Mato Grosso, and suggestions on oscine-suboscine relationships. Acta Amaz. 1976;6(3):379-94.

Willis EO. The composition of avian communities in remanescent woodlots in southern Brazil. Pap Avulsos Zool. 1979;33(1):1-25. 